\title{
Search and selection processes in implicit and explicit word-stem completion performance in young, middle-aged, and older adults
}

\author{
LEE RYAN \\ University of Arizona, Tucson, Arizona \\ ARNE OSTERGAARD and LAUREN NORTON \\ University of California, San Diego, California \\ and \\ JENNIFER JOHNSON \\ University of Arizona, Tucson, Arizona
}

\begin{abstract}
In the present investigation, we sought to (1) replicate previous reports of impaired word-stem completion (WSC) priming and cued recall performance in older adults, (2) gain a better understanding of the differential roles of search and selection processes in implicit and explicit WSC, and (3) determine the particular aspects of WSC performance that are influenced by age. Experiment 1 demonstrated that older adults primed and recalled fewer items on a WSC task than middle-aged adults did, who in turn primed and recalled fewer itmes than young adults did. A series of item analyses indicated that choice of a stem completion is influenced by two independent sources of information: the familiarity of the completion reflected by word frequency and the cue-specific match between the stem and the completion word based on the common pronunciation of the stem. Experiment 2 demonstrated that older adults utilized cue-specific matching to the same degree as young and middle-aged adults. However, the impact of target familiarity changed across the lifespan. Older adults produced and recalled high-frequency targets as well as young adults but produced and recalled fewer low-frequency targets than did young participants. The results are consistent with the view that older adults rely on familiarity to a greater degree than do young adults.
\end{abstract}

Considerable effort has been made to characterize the memory changes evident in older adults. An intriguing finding has been that not all memory tasks are affected equally. Several reviews have concluded that older adults are impaired on explicit tasks, such as free recall or cued recall, whereas implicit task performance, such as lexical decision or word identification, remains relatively intact (Howard, 1988; Parkin, 1993). However, the dissociation between implicit and explicit memory performance in older adults is not always so consistent. Although still in the minority, a growing number of studies have demonstrated decreased implicit memory (or item priming) in older adults (for reviews, see Fleischman \& Gabrieli, 1998; Howard, 1988; Light \& Lavoie, 1993).

Whether or not age differences in priming occur may depend primarily on the choice of the priming task. At least

This research was supported by Grant AG06849 from the National Institute on Aging to A.O. and by a National Science and Engineering Research Council Postdoctoral Fellowship to L.R. Our thanks to Morris Moscovitch and Elizabeth Glisky for their comments and suggestions and to Peter Graf and our reviewers for their constructive criticism. Correspondence concerning this article should be addressed to L. Ryan, Department of Psychology, University of Arizona, Tucson, AZ, 85721-0068 (e-mail: ryant@u.arizona.edu). five investigations using word-stem completion (WSC) have found decreased priming in older adults when compared with young adults (Chiarello \& Hoyer, 1988; Davis et al., 1990; Hultsch, Masson, \& Small, 1991; Schacter, Church, \& Osowiecki, 1994, Experiment 1; Small, Hultsch, \& Masson, 1995; Winocur, Moscovitch, \& Stuss, 1996), although at least an equal number of studies have found no age-related differences in priming (Dick, Kean, \& Sands, 1989; Java \& Gardiner, 1991; Light \& Singh, 1987; Park \& Shaw, 1992; Schacter et al., 1994, Experiment 2). In contrast, reliable age differences in priming are consistently absent in word-fragment completion (Light, Singh, \& Capps, 1986; Rybash, 1994; Winocur et al., 1996), lexical decision (Balota \& Ferraro, 1996; Karayanidis, Andrews, Ward, \& McConaghy, 1993), and word identification (Hashtroudi, Chrosniak, \& Schwartz, 1991; Light \& Kennison, 1996; Light, LaVoie, ValenciaLaver, Albertson-Owens, \& Mead, 1992; Light \& Singh, 1987; but see Abbenhuis, Raajmakers, Raajmakers, \& Van Woerden, 1990).

Why is WSC more sensitive than other tasks to agerelated differences in verbal priming? A number of researchers have noted that WSC behaves differently from such tasks as fragment completion or word identification 
(Graf \& Mandler, 1984; Nelson, Canas, Bajo, \& Keelean, 1987; Postle \& Corkin, 1999; Squire, Shimamura, \& Graf, 1987; but see Roediger, Weldon, Stadler, \& Riegler, 1992). The difference may be due to the prominent role of search and selection processes in WSC. Winocur et al. (1996) posited a model of WSC in which the stem initiates a search through the lexicon. Multiple matches are generated on the basis of the perceptual overlap between the cue and the lexical items, from which a single response is then selected. In contrast, tasks such as fragment completion or word identification do not initiate search through the lexicon or require selection among competing alternatives. The cues presented in these tasks often have a single solution or, at best, are poor cues for generating multiple matches and thus act as a partial re-presentation of the perceptual record (see also Graf \& Mandler, 1984; Humphreys, Bain, \& Pike, 1989).

Several accounts of memory impairment in older adults have focused on the role of search and selection. According to Winocur and his colleagues, older participants perform poorly on WSC because of inefficient search strategies that decrease the utility of a perceptual cue in defining a set of possible target items (Winocur et al., 1996). Supporting this hypothesis, Davis et al. (1990) found that lower WSC priming in older adults was associated with poor performance on the Wisconsin Card Sorting Test, a test of rule learning that requires strategy, flexibility, and inferential learning (see also Winocur et al., 1996).

In an alternative account, Balota and Ferraro (1993, 1996) have argued that memory decline in older adults is due to the loss of inhibitory control over partially activated information, an idea similar to one proposed earlier by Hasher and Zacks (1988). The loss of inhibition results in highly familiar or automatic responses intervening during normal task processes. Balota and Ferraro (1996) reported that older adults were more sensitive to word frequency on a lexical decision task; older adults showed a greater performance difference between high- and lowfrequency words, as compared with young adults. On WSC, the increased propensity in older adults to produce high-frequency items may override the effect of a single prior presentation, thereby decreasing priming, particularly when the target word is low frequency. It is unclear, however, whether the same frequency effect should be present when the task is explicit recollection.

In order to understand the mechanism of memory impairment in older adults, the critical information will likely be the degree to which various factors predict performance on a task, rather than absolute differences in performance between younger and older adults. Differentiating between the alternative views described above, for example, requires independent measurement of the degree to which familiarity and search processes are utilized in WSC priming and cued recall in younger and older adults. Although there is little to suggest how search is accomplished, the impact of familiarity in WSC is well known. Postle and Corkin (1999) recently showed that familiar- ity with the target word, as reflected in word frequency, influenced WSC completion rates. High-frequency words were more likely to be produced at baseline and also obtained more priming and higher rates of cued recall when compared with low-frequency words. Graf and Williams (1987) considered the influence of familiarity on baseline WSC performance and found that word frequency, word length, and the number of meanings per target item were predictors for the choice of a first completion for a stem; the more familiar an item, the more likely it was to be chosen. However, the combination of these factors accounted for a surprisingly small amount of baseline variance, and the authors concluded that the choice of a first completion must be constrained largely by factors having to do with the nature of the individual word-stem cue. They did not speculate as to what these cue-specific factors might be.

The goals of the present investigation were to understand more about search and selection in WSC, whether these factors influence priming and cued recall similarly, and whether utilization of these factors differs in younger and older adults. Our working hypothesis was that the selection of a stem completion is based on two independent sources of information. First, completion probability increases with familiarity, as reflected by word frequency, word length, and number of meanings per target item, variables that are highly intercorrelated (Balota \& Chumbley, 1984; Graf \& Williams, 1987). Second, we suggest that WSC search is driven by a cue-specific matching process between the phonological properties of the stem and the target word. Individuals adopt a strategy of pronouncing the stem internally and then search for items in the lexicon that begin with a similar sound. A recent study by Rueckl and Mathew (1999) highlights the importance of phonology for WSC priming by demonstrating that target words can be primed by their homophone (e.g., week primes the stem WEA__ for the target weak). We extend this notion by proposing that the way a participant pronounces a stem cue will be an important determinant of the choice of a completion and will influence the probability that a particular target is primed or recalled.

Two experiments are presented here. In Experiment 1, we sought to replicate the finding previously reported in the literature of decreased WSC priming and cued recall in older adults and to provide a database for a series of item analyses that elucidate the roles of familiarity and cue-specific search factors in WSC. Experiment 2 assessed whether or not familiarity and cue-specific search are utilized to the same degree by young, middle-aged, and older adults.

\section{EXPERIMENT 1}

As was noted earlier, results thus far are about evenly split on the question of whether WSC priming does or does not decrease in older adults. The inability in some studies to detect subtle group differences for WSC may 
be due to a lack of power (Lavoie \& Light, 1994). Power is influenced not only by the number of participants that are tested, but also by task difficulty. On some priming tasks, poorer levels of baseline performance are associated with larger priming effects, as is the case with perceptual identification (Jernigan \& Ostergaard 1993; Ostergaard, 1994, 1998, 1999). Ostergaard (1998) found that experimental variables such as word frequency and the length of delay between study and test had a significant effect on priming, but only under difficult word identification conditions that produced relatively large priming effects. Under easy conditions, in which baseline identification was fast and priming effects were small, none of the variables significantly affected priming. For WSC, the relationship between baseline completion probability and priming is unclear. Baseline probability may reflect difficulty in accessing the long-term representation of the target. In that case, one might expect to see that decreases in baseline completion probability will result in increased priming. Materials for Experiment 1 included target items at three levels of baseline completion probability that were predetermined by a large normative data set available in our laboratory (see the Method section), ranging from moderately high probability to very rare completions. We expected that the largest differences in performance between older and younger adults would be evident under baseline conditions that maximized priming.

Experiment 1 tested young, middle-aged, and older adults on WSC with either implicit or explicit instructions. Since most research in this area has compared only young and older adults, it is unclear whether changes in WSC performance occur across the entire lifespan or whether the decline is best described as a threshold ef- fect, with performance dropping off only in elder years. The inclusion of a middle-aged group allowed this issue to be addressed directly.

\section{Method}

Participants. The participants $(N=150$; see Table 1$)$ were recruited through two separate sources. Participants under the age of 55 were recruited through advertisements posted on the University of California at San Diego (UCSD) campus and in local newspapers. Participants over the age of 55 were recruited from the pool of normal control participants at the Alzheimer's Disease Research Center (ADRC) at UCSD. Volunteers were tested individually in a single session lasting approximately one half hour and were paid $\$ 5$ for their participation. Three age groups were tested; young $(n=$ 50 ; range, 18-35 years of age), middle-aged ( $n=50$; range 3655 years of age), and older ( $n=50$; range, $56-88$ years of age) adults. Within each age group, 30 volunteers were randomly assigned to the implicit condition, and the remaining 20 participants were assigned to the explicit condition. Groups were well matched on education $(F<1)$, and there were no interactions between age group and test instruction or between education level and test instruction $(F \mathrm{~s}<1)$.

Materials. Word stems and targets were chosen from a large normative baseline data set for 99 three-letter stems collected from 50 young (ages, 18-35), 50 middle-aged (ages, 36-55), and 50 older (ages, 56-85) volunteers. The participants in the normative study were given a booklet containing 99 stems, each followed by four blank spaces. They were instructed to write down the first four stem completions that came to mind but to refrain from using foreign words, proper names, or repetitions of the same word with multiple endings.

Forty-eight three-letter word stems were chosen for the present study from the normative baseline (NB) data set collapsed across all 150 participants (ages, 18-85). For each stem, three target solutions were chosen that differed in baseline probabilities for the first completion and across four completions. High-NB targets had a moderate first probability (mean, $.25 ; S D, .11$ ), and a moderate to high fourcompletions probability (mean, .51: $S D, .15$ ). Medium- $N B$ targets had a low first probability (mean, .06; $S D, .04$ ) and a consistently

Table 1

Mean Age and Education (in Years) for Young, Middle-Aged, and Older Adults in Experiments 1 and 2

\begin{tabular}{|c|c|c|c|c|c|c|}
\hline \multirow[b]{2}{*}{ Group } & \multicolumn{3}{|c|}{ Age } & \multicolumn{3}{|c|}{ Education } \\
\hline & $M$ & $S D$ & Range & $M$ & $S D$ & Range \\
\hline \multicolumn{7}{|c|}{ Experiment 1} \\
\hline \multicolumn{7}{|c|}{ Implicit condition } \\
\hline Young & 23.5 & 4.1 & $18-35$ & 15.0 & 1.5 & $13-18$ \\
\hline Middle & 46.0 & 6.4 & $36-55$ & 14.8 & 2.5 & $11-21$ \\
\hline Older & 73.2 & 7.3 & $59-84$ & 15.1 & 2.9 & $8-20$ \\
\hline \multicolumn{7}{|c|}{ Explicit condition } \\
\hline Young & 25.5 & 4.9 & $20-35$ & 15.4 & 1.4 & $12-18$ \\
\hline Middle & 44.8 & 4.9 & $36-53$ & 14.7 & 2.4 & $10-21$ \\
\hline Older & 71.5 & 7.1 & $56-88$ & 14.8 & 2.7 & $9-20$ \\
\hline \multicolumn{7}{|c|}{ Experiment 2} \\
\hline \multicolumn{7}{|c|}{ Implicit condition } \\
\hline Young & 24.4 & 5.9 & $16-34$ & 14.4 & 2.7 & $10-19$ \\
\hline Middle & 45.2 & 6.1 & $36-55$ & 16.3 & 2.1 & $11-20$ \\
\hline Older & 65.2 & 6.0 & $56-75$ & 14.9 & 2.2 & $12-20$ \\
\hline \multicolumn{7}{|c|}{ Explicit condition } \\
\hline Young & 25.3 & 4.5 & $18-34$ & 15.4 & 2.1 & $10-20$ \\
\hline Middle & 44.5 & 5.3 & $36-55$ & 16.2 & 1.8 & $11-20$ \\
\hline Older & 68.5 & 5.4 & $57-77$ & 15.8 & 2.3 & $11-20$ \\
\hline
\end{tabular}


higher four-completions probability (mean, .32; $S D, .11$ ). The low$N B$ targets were rare, with a low probability either for first completion (mean, .02; $S D, .02$ ) or across four completions (mean, .09; $S D$, $.04)$. Solutions were chosen not to produce three nonoverlapping NB categories, but to fit the relative pattern of baseline probabilities. As an example, for the stem RET, retire was chosen as the highNB target (first $=.25$, four $=.43$ ), retain was chosen as the mediumNB target (first $=.08$, four $=.27$ ), and retract was chosen as the low-NB target (first $=.01$, four $=.05$ ).

Stems were randomly divided into two sets of 24 . Six study lists were constructed, three for each of the two stem sets. A study list contained four filler items followed by 24 targets, with one target word for each stem, including 8 high-, 8 medium-, and 8 low-NB targets. The six study lists were counterbalanced across age groups and test instruction conditions.

Procedure. The participants were told that the experiment included several tasks concerning language and the perception of words. During study, the participants were shown a study list. Words were presented one at a time on a computer screen, in random order, for $2 \mathrm{sec}$, followed by a blank screen for $2 \mathrm{sec}$. The participants were instructed to read each word aloud and to rate each word for "pleasantness" on a scale of 1 (very unpleasant) to 5 (very pleasant). The participants in all age groups had no difficulty rating each word well within the 4-sec time allotted. After the rating task, the experimenter engaged the participant in conversation for $5 \mathrm{~min}$.

During the test phase that followed, 48 stems were presented on a computer screen in one of four fixed random orders. Twenty-four of the stems could be completed with a target word, whereas the other 24 stems provided baseline completion probabilities. Thirty participants from each age group were instructed to complete the stem with the first word that came to mind. The remaining 20 participants were instructed to complete each stem with a word that they saw during the pleasantness-rating task. If a participant could not recall a word from the list, they were instructed to respond with the first word that came to mind. The participants in both test instruction conditions were required to respond to every word stem.

\section{Results}

Implicit performance. Baseline and priming data were analyzed separately with a mixed-factor analysis of variance (ANOVA) that included age group (young, middle-aged, or older), and NB level (high, medium, or low) as the repeated measure (see Table 2). Baseline data showed a significant main effect for NB level $[F(1,87)=$ 530.87, $\left.M S_{\mathrm{e}}=0.003, p<.0001\right]$. Follow-up paired $t$ tests indicated that high-NB targets were produced more often than medium-NB targets $[t(89)=14.47, p<.0001]$ and medium-NB targets were produced more often than low-NB targets $[t(89)=8.16, p<.0001]$. However, there was no indication of a difference in baseline performance across the age groups or of an interaction between age group and NB level $\left(F_{\mathrm{S}}<1\right)$.

Priming scores were calculated for an individual at each of the three NB levels by subtracting the individual's baseline performance for a given NB level from the probability of producing a previously studied target item for that NB level. The significant main effect of NB level $\left[F(1,87)=22.54, M S_{\mathrm{e}}=0.027, p<.0001\right]$ indicated that priming differed across NB levels. Follow-up paired $t$ tests showed greater priming for high-NB targets than for medium-NB targets $[t(89)=4.18, p<.001]$, whereas there was no difference between medium- and low-NB targets $(t<1)$. Importantly, there was also a main effect of age group $\left[F(2,87)=15.51, M S_{\mathrm{e}}=0.042, p<.0001\right]$. Least significant difference (LSD) follow-up tests indicated that young participants obtained more priming than did middle-aged participants, who in turn obtained more priming than did older participants $(p \mathrm{~s}<.05)$. Age did not interact with NB level $[F(2,87)=1.52$, n.s.]. We also calculated the linear correlation between priming and age for the entire group, using a single priming score computed by collapsing across all three NB levels. The scatterplot shown in Figure 1 suggests that priming scores decreased linearly across the entire age range (Pearson $r=$ $.51, p<.01)$. A polynomial regression analysis did not yield significant quadratic or higher order components, confirming the linearity of the relationship.

Explicit performance. Since the participants were required to respond to every stem, probably not all the studied targets that were produced were due to conscious recollection of the item but, instead, may have been produced

Table 2

Mean Completion Probabilities for Implicit and Explicit Word-Stem Completion in Experiment 1 at Three Normative Base Rate (NB) Levels

\begin{tabular}{|c|c|c|c|c|c|c|c|c|c|}
\hline \multirow[b]{2}{*}{ NB Level } & \multirow[b]{2}{*}{ Condition } & \multicolumn{2}{|c|}{ Young } & \multicolumn{2}{|c|}{ Middle-aged } & \multicolumn{2}{|c|}{ Older } & \multicolumn{2}{|c|}{ All Subjects } \\
\hline & & $M$ & $S D$ & $M$ & $S D$ & $M$ & $S D$ & $M$ & $S D$ \\
\hline \multicolumn{10}{|c|}{ Implicit } \\
\hline \multirow[t]{2}{*}{ High } & Baseline & .20 & .08 & .21 & .07 & .22 & .09 & .21 & .08 \\
\hline & Priming & .38 & .21 & .26 & .21 & .24 & .23 & .29 & .22 \\
\hline \multirow[t]{2}{*}{ Medium } & Baseline & .08 & .03 & .05 & .04 & .06 & .06 & .06 & 05 \\
\hline & Priming & .26 & .21 & .18 & .17 & .11 & .15 & .19 & .19 \\
\hline \multirow[t]{2}{*}{ Low } & Baseline & .02 & .03 & .02 & .02 & .01 & .02 & .02 & .02 \\
\hline & Priming & .27 & .14 & .18 & .17 & .07 & .11 & .17 & .16 \\
\hline \multirow[t]{2}{*}{ Total priming } & & .31 & .12 & .21 & .11 & .14 & .12 & & \\
\hline & & & licit & & & & & & \\
\hline \multirow[t]{2}{*}{ High } & Baseline & .22 & .07 & .25 & .07 & .27 & .09 & .25 & .08 \\
\hline & Cued recall & .44 & .24 & .33 & .20 & .26 & .20 & .34 & .22 \\
\hline \multirow[t]{2}{*}{ Medium } & Baseline & .08 & .05 & .08 & .05 & .05 & .05 & .07 & .05 \\
\hline & Cued recall & .28 & .28 & .34 & .15 & .21 & .15 & .27 & .19 \\
\hline \multirow[t]{2}{*}{ Low } & Baseline & .02 & .03 & .01 & .02 & .02 & .03 & .02 & .03 \\
\hline & Cued recall & .37 & .19 & .30 & .20 & .12 & .15 & .26 & .21 \\
\hline Total cued recall & & .36 & .18 & .32 & .15 & .20 & .13 & & \\
\hline
\end{tabular}



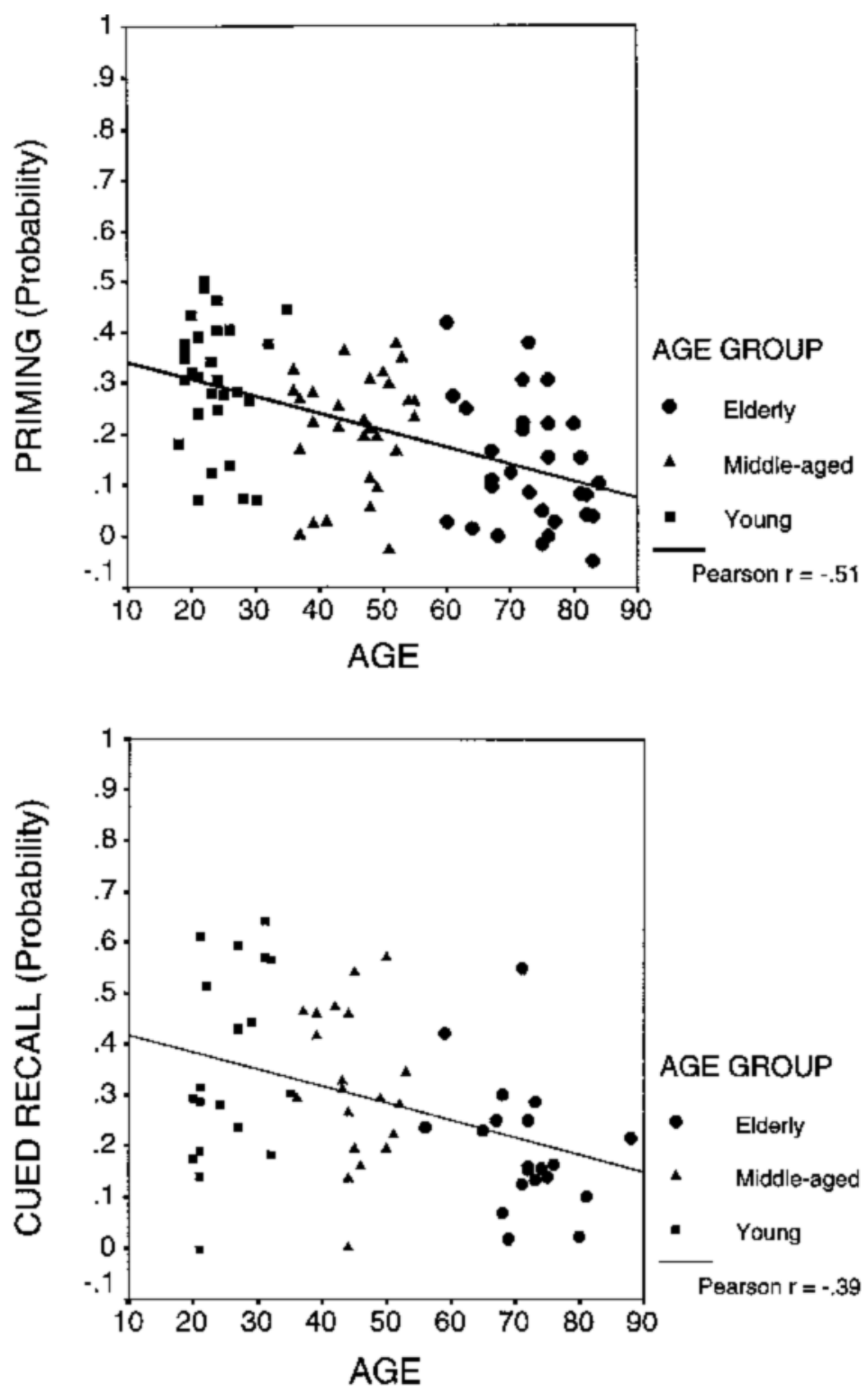

Figure 1. Scatterplots depicting the overall probability of priming and cued recall (corrected for baseline performance) plotted against age for the participants in Experiment 1.

as a result of priming. Cued recall performance was therefore analyzed in a manner consistent with priming, comparing first baseline completion probabilities, and then calculating cued recall scores at each of the three NB levels by subtracting the baseline probability from the probability of recalling a previously studied target item. The results are listed in Table 2.

Similar to the implicit results, the number of targets produced at baseline differed for the three NB levels $\left[F(1,57)=497.37, M S_{\mathrm{e}}=0.003, p<.0001\right]$. Paired $t$ tests indicated that high-NB targets were produced more often than medium-NB targets $[t(59)=15.50, p<.0001]$ and that medium-NB targets were produced more often than
low-NB targets $[t(59)=6.65, p<.0001]$. However, there was no difference in baseline performance across the age groups $(F<1)$ and no interaction between age group and NB level $\left[F(2,57)=2.57, M S_{\mathrm{e}}=0.003\right.$, n.s. $]$.

Cued recall scores, corrected for baseline probability, indicated that recall performance depended on the NB level $\left[F(1,57)=9.03, M S_{\mathrm{e}}=0.021, p<.01\right]$. Paired $t$ tests showed that cued recall was greater for high-NB targets than for medium-NB targets $[t(59)=2.01, p<$ $.05]$ but that there was no difference in recall for mediumand low-NB targets $(t<1)$. Cued recall performance also differed with age $\left[F(2,57)=5.86, M S_{\mathrm{e}}=0.072, p<.01\right]$, but age did not interact with NB level. LSD tests indicated 
that older participants recalled fewer items than did either young or middle-aged participants $(p s<.05)$ but that young and middle-aged participants recalled similar numbers of target items. However, with a single corrected cued recall score collapsed across the three NB levels, the correlation between age and cued recall yielded a Pearson $r$ of $-.39(p<.01$; see Figure 1$)$. Using a recall score uncorrected for baseline performance yielded a similar $r$ value $(r=-.41, p<.01)$. This relationship was best described as linear, since a polynomial regression did not indicate significant higher order components.

\section{Discussion}

The influence of baseline in WSC performance. Significant priming was obtained at all three NB levels, even for target words that are rarely produced as stem completions, and the higher the likelihood that a target solution was generated at baseline, the larger the priming effect. This pattern is opposite to that seen on priming tasks such as lexical decision or perceptual identification, where lower levels of baseline performance are associated with larger priming effects (Ostergaard, 1998; Scarborough, Cortese, \& Scarborough, 1977). On lexical decision and word identification tasks, there is a single correct response for each item. Baseline performanceoften measured in reaction time-will reflect the fluency or ease with which a decision is made for that particular item. The more difficult the decision, the more likely it is that a single study episode will have an appreciable impact on performance. In contrast, WSC does not constrain the response to a single correct item (Fleischman \& Gabrieli, 1998) but, instead, instructs the participant to select the item that is most readily accessible from among many possible solutions. Studying a target increases accessibility, in comparison with competing solutions, thereby increasing the likelihood that the target will be produced. A very low baseline probability indicates that other stem solutions are considerably more accessible, making it unlikely that a single presentation of the weak solution will be sufficient to overcome the preexperimental differences in accessibility. On the other hand, for items with higher baseline probabilities, a single study episode may result in making the target consistently more accessible, as compared with competing alternatives.

Age effects in WSC priming and cued recall. Although baseline completion probabilities remained stable, priming and cued recall performance differed in all three age groups. One other WSC priming study (Davis et al., 1990) tested participants from the 2 nd to the 8th decade and found that priming scores decreased significantly only within the 7 th and 8 th decades. The authors attributed the absence of a decline in the 6th decade to a lack of power, drawing attention to the fact that the priming scores for this group fell midway between the scores for the youngest and the oldest participants. Their Figure 3 (Davis et al., 1990, p. 294) shows that the mean priming scores for participants in the 4 th and 5 th decades also fell midway between those for the youngest and the oldest groups and at about the same level as that for the participants in the 6th decade. Taken together, these studies argue against a threshold effect, according to which WSC performance declines only in the late decades of life.

A concern in the present study was the degree to which age group differences in priming arose because priming was "contaminated" by the participants' use of an explicit strategy. Instead of producing the first word that came to mind, the participants may have completed at least some stems with a word they had seen previously. Age differences may arise because younger participants are more likely than older adults to adopt an explicit strategy, thereby increasing their performance disproportionately to that of older adults. We did not directly ask the participants in the priming condition whether they recognized that they were producing previously studied items. Although this perception by itself has been shown to have no effect on the pattern of performance on word-stem completion tests (Bowers \& Schacter, 1990; RichardsonKlavehn \& Bjork, 1988), it would be useful to have more direct evidence. In the next experiment we address this issue. We assume that both tests, implicit and explicit, utilize processes that are necessary for the other type of test to some degree (Jacoby, 1991; Johnson \& Hasher, 1987), but it remains possible that one age group used an explicit strategy to a different degree than did another age group.

\section{ITEM ANALYSES}

The purpose of the item analyses was to identify familiarity and cue-specific factors that guide search and selection in WSC. It is well documented that items can behave differently on the explicit and the implicit forms of a task (Hintzman \& Hartry, 1990). Thus, we considered whether search and selection factors differentially influence baseline, priming, and cued recall.

Two variables associated with the familiarity of the target word were considered: the absolutefrequency (AF) of the word, and the rank order frequency (RF). AF refers to the frequency of occurrence of a word within a corpus of written or spoken language, such as the normative data set compiled by Kučera and Francis (1967). However, in WSC, AF may not be as important as the frequency of a word, relative to other solutions for the particular stem. That is, when all possible completions for a stem are rank ordered on the basis of word frequency, the most common solution may be chosen most often. Although AF and RF will correlate to some degree, two words with considerably different AFs both may be the highest frequency completion for their respective stems and may thus be equally likely to be produced at baseline.

Turning to cue-specific factors, we hypothesized that participants adopt a strategy of pronouncing the stem internally and then search for items in the lexicon that begin with a similar sound. For stems with a common pronunciation, a subset of completions will be produced 
more consistently. Thus, in response to the stem BAR, participants will be more likely to produce the words bark or barter rather than barren. In addition, participants probably perceive the stem as a single syllable or unit and will be more likely to choose completions that maintain that stem as a separate and intact unit. In response to the stem COU, participants may be more likely to produce the word cougar than words like coupe or cough. Two cue-specific factors were considered: the pronunciation match between the stem and the beginning of the target word (sound match) and the number of syllables in the target word (syllable length).

The target words in Experiment 1 were chosen solely on the basis of their baseline probabilities and without regard to frequency or cue-specific factors. In the following item analyses, completion probabilities for each item were calculated for implicit baseline, implicit studied, explicit baseline, and explicit studied, collapsing across three age groups.

\section{Word Frequency}

AF counts for targets (Kučera \& Francis, 1967) ranged from 1 to 796, with a median frequency of 15.5 that was used as the criterion for separating targets into high- and low-frequency categories. RF was determined from the normative data set (see the Method section, Experiment 1). Extremely rare solutions were excluded (completions produced by fewer than 3 of 150 participants). For a given stem, all completions were rank ordered on the basis of AF, with 1 representing the highest frequency item. Targets from Experiment 1 were then coded for RF and ranged from 1 to 29, with a median rank order frequency of 4.5 that was used as the criterion for separating targets into high and low rank order frequency categories. Note that an RF of 1 indicates the highest frequency word that can complete the stem; it does not necessarily correspond to the item with the highest baseline probability for that stem.

Pearson correlation coefficients between AF, RF, and the four WSC measures are listed in Table 3 and were compared using Fischer's $z$ transformation tests. Correlations involving RF were consistently larger than those involving AF, and this difference was significant for explicit baseline (Fisher's $z=3.02, p<.01$ ) and implicit baseline $(z=2.42, p<.01)$. The correlation between RF and WSC measures depended on whether or not the items were pre-

Table 3

Pearson Correlation Coefficients Between Absolute Word Frequency (AF), Rank Order Word Frequency (RF), and Word-Stem Completion Performance Measures Under Implicit and Explicit Instruction Conditions

\begin{tabular}{ccccr}
\hline Condition & $\mathrm{AF}$ & $p$ & $\mathrm{RF}$ & \multicolumn{1}{c}{$p$} \\
\hline Implicit & & & & \\
Baseline & .10 & n.s. & -.37 & .0001 \\
Priming & .16 & .06 & -.19 & .03 \\
Explicit & & & & \\
Baseline & .10 & n.s. & -.43 & .0001 \\
Cued recall & .18 & .03 & -.23 & .006 \\
\hline
\end{tabular}

viously studied. RF correlated more strongly with baseline than with studied probability for both explicit items $(z=1.90, p<.05)$ and implicit items $(z=1.65, p<.05)$.

\section{Cue-Specific Variables and the Relationship to Frequency}

Sound match (matched or mismatched) and syllable length (single or multiple) were considered in separate item analyses. Words were also classified as high or low RF, creating two between-items factors, the cue-specific factor crossed with RF (high vs. low), and two withinitems factors, study condition (baseline vs. studied) and test instruction (implicit vs. explicit). For brevity, only those effects involving the cue-specific variable, RF, and their interactions will be discussed.

Sound match. Twelve volunteers were asked to read aloud a list of 48 stems as they would be pronounced if the stem was a word. A stem was considered to have a common pronunciation if 8 of 12 volunteers pronounced the stem similarly. Stems without a common pronunciation $(n=7)$ and their corresponding target items were excluded. As an example, 11 of 12 participants pronounced the stem SAL as in the word salad, even though the stem can be pronounced as in the words sale or salt. In contrast, 5 participants pronounced the stem MEA as in the word mean, another 4 participants pronounced it as in measure, and 3 participants pronounced it as in meander. Stems were also excluded if there was only one possible pronunciation $(n=10$; e.g., the stem COR is pronounced the same for all possible completions in the English language, as in the word correct). The remaining 31 stems and 93 corresponding targets (3 for each stem) were coded for sound match. A target was coded as matched when the beginning of the target word was pronounced similarly to the stem or as mismatched when it was pronounced differently. Sound match judgments were made by two independent raters, who agreed on $98 \%$ of the targets. For the few remaining words, classifications were agreed upon by discussion. Items were classified into four sound-match-RF conditions: matched-high $(n=23)$, mismatched-high $(n=22)$, matched-low $(n=25)$, and mismatched-low $(n=23)$.

The results (see the top part of Table 4) indicated that matched items obtained overall higher completion probabilities than did mismatched items $[F(1,89)=11.99$, $\left.M S_{\mathrm{e}}=0.112, p<.001\right]$. High-RF targets also produced higher completion rates, as compared with low-RF targets $\left[F(1,89)=12.61, M S_{\mathrm{e}}=0.112, p<.001\right]$. Sound match and RF did not interact, suggesting that these two factors influenced performance independently of one another $(F<1)$. Sound match did not differ under implicit and explicit test instructions $\left[F(1,89)=2.74, M S_{\mathrm{e}}=0.011\right.$, n.s.] but interacted significantly with study condition $\left[F(1,89)=8.92, M S_{\mathrm{e}}=0.022, p<.01\right]$. The difference between matched and mismatched items was greater when the item was previously studied than when it was not. A significant sound match difference was indicated by $t$ tests for both previously studied items [mean, .44 vs. 
Table 4

Item Analysis Results Involving Two Cue-to-Target Variables and Rank Order Word Frequency (RF)

\begin{tabular}{|c|c|c|c|c|c|c|c|c|c|c|c|c|}
\hline \multirow[b]{3}{*}{ Condition } & \multicolumn{6}{|c|}{ Studied } & \multicolumn{6}{|c|}{ Nonstudied } \\
\hline & \multicolumn{2}{|c|}{ Matched } & \multicolumn{2}{|c|}{ Mismatch } & \multirow[b]{2}{*}{$M$} & \multirow[b]{2}{*}{$S D$} & \multicolumn{2}{|c|}{ Matched } & \multicolumn{2}{|c|}{ Mismatch } & \multirow[b]{2}{*}{$M$} & \multirow[b]{2}{*}{$S D$} \\
\hline & $M$ & $S D$ & $M$ & $S D$ & & & $M$ & $S D$ & $M$ & $S D$ & & \\
\hline & \multicolumn{10}{|c|}{ Sound Match (Matched or Mismatch) Versus RF (High or Low) } & & \\
\hline \multicolumn{13}{|l|}{ Implicit } \\
\hline High RF & .43 & .29 & .31 & .24 & .36 & .27 & .19 & .19 & .12 & .14 & .16 & .17 \\
\hline Low RF & .34 & .25 & .16 & .11 & .25 & .22 & .06 & .08 & .03 & .05 & .04 & .07 \\
\hline Combined RF & .38 & .27 & .23 & .20 & & & .12 & .16 & .07 & .11 & & \\
\hline \multicolumn{13}{|l|}{ Explicit } \\
\hline High RF & .53 & .30 & .37 & .31 & .46 & .30 & .27 & .15 & .11 & .12 & .20 & .21 \\
\hline Low RF & .45 & .19 & .26 & .16 & .35 & .21 & .06 & .08 & .03 & .07 & .05 & .07 \\
\hline Combined RF & .49 & .25 & .31 & .25 & & & .17 & .21 & .07 & .10 & & \\
\hline \multicolumn{13}{|c|}{ Syllable Length (Single or Multiple) Versus RF (High or Low) } \\
\hline \multicolumn{13}{|l|}{ Implicit } \\
\hline High RF & .44 & .27 & .29 & .22 & .37 & .15 & .20 & .19 & .09 & .10 & .15 & .16 \\
\hline Low RF & .27 & .20 & .15 & .23 & .16 & .21 & .04 & .05 & .04 & .04 & .04 & .04 \\
\hline Combined RF & .36 & .24 & .27 & .22 & & & .12 & .13 & .07 & .07 & & \\
\hline \multicolumn{13}{|l|}{ Explicit } \\
\hline High RF & .52 & .34 & .38 & .26 & .45 & .30 & .25 & .24 & .11 & .11 & .18 & .17 \\
\hline Low RF & .39 & .19 & .31 & .22 & .35 & .20 & .05 & .07 & .04 & .05 & .05 & .07 \\
\hline Combined RF & .46 & .26 & .35 & .24 & & & .15 & .15 & .08 & .08 & & \\
\hline
\end{tabular}

$.27 ; t(91)=3.53, p<.001]$ and baseline items [mean, .14 vs. $.07 ; t(91)=2.29, p<.05]$. No higher order interactions approached significance.

Syllable length. Targets were coded as single or multiple syllable. Three stems were excluded because the stem contained two syllables, making a single syllable completion impossible (e.g., ABO). Another eight stems were excluded because no single-syllable completion existed in the English language (e.g., OBS or APP). Items were classified into four syllable-length-RF conditions: singlehigh $(n=33)$, multiple-high $(n=22)$, single-low $(n=$ $23)$, and multiple-low $(n=33)$.

Multiple-syllable items (see the bottom part of Table 4) obtained higher completion probabilities than did single-syllable items $\left[F(1,107)=7.92, M S_{\mathrm{e}}=0.10, p<\right.$ $.01]$, and there was also a main effect of $\operatorname{RF}[F(1,107)=$ $\left.13.87, M S_{\mathrm{e}}=0.10, p<.001\right]$. No interactions approached significance.

\section{Discussion}

Determinants of WSC. The choice of a completion in WSC is determined both by the frequency of the completion word and by several cue-specific factors. The critical frequency variable in WSC appears to be the RF of a word in comparison with its competitor completions, rather than the absolute frequency of a word in the English language. Higher RF items generally have higher probabilities of being chosen as a completion. RF predicts baseline probabilities more than studied probabilities, suggesting that recent exposure to a target changes the familiarity or effective frequency of the word and that, therefore, preexperimental RFs will be less predictive of completion rates.

The results highlight the importance of phonology in stem completion. Participants utilize the sound of the stem to guide search through the lexicon, and thus the selected completion is likely to be a word that matches the most typical pronunciation of the stem but will also keep the stem intact as a separate syllable unit. In the present study, we identified two variables that are consistent with this general hypothesis, although no doubt others exist as well.

\section{EXPERIMENT 2}

Experiment 2 determined whether familiarity and cuespecific matching variables can account for the decline in WSC priming and cued recall performance in older adults. We also assessed the impact of participants' awareness regarding the nature of the implicit test on priming performance. Targets were chosen that varied in RF and sound match. We chose sound match as the cue-specific variable because it most directly reflected the use of pronunciation for search.

\section{Method}

Participants. Participants $(N=180$; see Table 1$)$ from three age groups were tested; young ( $n=60$; range, 16-35), middle-aged $(n=60$, range, 36-55), and older ( $n=60$, range, 56-77) adults. Half of the participants ( 30 from each age group) were randomly assigned to the implicit test condition, whereas the remainder were assigned to the explicit test condition. Separate ANOVAs on years of education and age indicated that the groups were well matched on years of education and that the groups were matched in age and education levels across test instruction conditions (all $F \mathrm{~s}<1$ ).

Materials. Stems (48) were chosen with a single target for each stem. Targets included an equal numbers of high-RF (mean, 2.0; $S D, .9$; range, 1-3) and low-RF (mean, 7.2; SD, 3.1; range, 4-16) items. Within each RF category, equal numbers of targets were sound matched and mismatched. Two equivalent study lists of 24 targets were created, which were presented equally often at study and test to provide baseline completion rates.

Procedure. The study and test procedures were identical to those in Experiment 1. After completing the WSC task, the participants in the implicit condition were asked about their perception of the 
Table 5

Mean Completion Probabilities for Word-Stem Completion in Experiment 2 for Targets That Vary in Sound Match (Matched or Mismatch) and Rank Order Frequency (High or Low)

\begin{tabular}{|c|c|c|c|c|c|c|c|c|c|}
\hline \multirow[b]{2}{*}{ Condition } & & \multicolumn{2}{|c|}{ Young } & \multicolumn{2}{|c|}{ Middle-aged } & \multicolumn{2}{|c|}{ Older } & \multicolumn{2}{|c|}{ All Subjects } \\
\hline & & $\bar{M}$ & $\overline{S D}$ & $M$ & $\overline{S D}$ & $\bar{M}$ & $\overline{S D}$ & $M$ & $S D$ \\
\hline \multicolumn{10}{|l|}{ Implicit } \\
\hline \multirow[t]{2}{*}{ Matched/high } & Baseline & .32 & .19 & .32 & .25 & .26 & .19 & .30 & .21 \\
\hline & Priming & .23 & .25 & .21 & .32 & .38 & .27 & .27 & .29 \\
\hline \multirow[t]{2}{*}{ Matched/low } & Baseline & .17 & .15 & .22 & .20 & .20 & .13 & .19 & .16 \\
\hline & Priming & .43 & .21 & .37 & .27 & .27 & .16 & .36 & .23 \\
\hline \multirow[t]{2}{*}{ Mismatch/high } & Baseline & .21 & .15 & .28 & .16 & .29 & .14 & .26 & .15 \\
\hline & Priming & .32 & .23 & .24 & .27 & .18 & .25 & .24 & .25 \\
\hline \multirow[t]{2}{*}{ Mismatch/low } & Baseline & .12 & .11 & .14 & .13 & .12 & .16 & .13 & .14 \\
\hline & Priming & .28 & .22 & .24 & .32 & .16 & .24 & .23 & .27 \\
\hline \multicolumn{10}{|l|}{ Explicit } \\
\hline \multirow[t]{2}{*}{ Matched/high } & Baseline & .39 & .23 & .37 & .21 & .38 & .24 & .38 & .23 \\
\hline & Cued recall & .28 & .27 & .26 & .29 & .30 & .36 & .28 & .31 \\
\hline \multirow[t]{2}{*}{ Matched/low } & Baseline & .22 & .14 & .31 & .14 & .27 & .20 & .27 & .16 \\
\hline & Cued recall & .46 & .23 & .32 & .22 & .31 & .25 & .36 & .24 \\
\hline \multirow[t]{2}{*}{ Mismatch/high } & Baseline & .33 & .18 & .28 & .16 & .31 & .16 & .30 & .17 \\
\hline & Cued recall & .24 & .26 & .32 & .30 & .21 & .20 & .26 & .26 \\
\hline \multirow[t]{2}{*}{ Mismatch/low } & Baseline & .17 & .14 & .15 & .18 & .18 & .15 & .16 & .16 \\
\hline & Cued recall & .41 & .27 & .27 & .30 & .25 & .22 & .31 & .27 \\
\hline
\end{tabular}

experiment, in order to ascertain whether or not they were aware that they had produced words that they had previously rated. No participant spontaneously described a connection between the pleasantness-rating task and WSC. When asked to guess how the two tasks might be connected, the most common response was that pleasant words might come to mind more readily than unpleasant words. A subgroup of the participants (9 young, 8 middle-aged, and 5 older adults) were aware that they had produced words they had previously rated for pleasantness. Implicit data were analyzed twice, once removing the aware participants and once including all the participants. Removing the data from the aware participants did not significantly alter the magnitude of the priming effects. The implicit analyses described below therefore include all the participants.

\section{Results}

Implicit WSC performance. The results of Experiment 2 are presented in Table 5. Baseline and priming scores were analyzed separately, using a mixed-factorial ANOVA with RF (high or low) and sound match (matched or mismatched) as within-subjects variables and age group (young, middle-aged, or older) as the between-subjects variable. At baseline, high-RF words were produced more often than low-RF words $\left[F(1,87)=41.73, M S_{\mathrm{e}}=0.03\right.$, $p<.0001]$, and matched words were produced more often than mismatched words $\left[F(1,87)=8.70, M S_{\mathrm{e}}=\right.$ $0.027, p<.01]$. The effects of word frequency and sound match did not interact with one another $(F<1)$. Baseline performance did not differ across age groups $[F(2,87)=$ $1.37, M S_{\mathrm{e}}=0.029$, n.s.], and age did not interact with either word frequency $(F<1)$ or sound match $(F<1)$. No other interactions approached significance.

Priming results (proportion of studied targets produced minus the proportion of baseline targets produced) indicated greater priming for matched than for mismatched items $\left[F(1,87)=9.38, M S_{\mathrm{e}}=0.062, p<.01\right]$. Sound match did not interact with age $(F<1)$. Although there was no overall priming difference between the age groups $\left[F(2,87)=1.84, M S_{\mathrm{e}}=0.093\right.$, n.s. $]$ and no main effect for $\operatorname{RF}\left[F(1,87)=2.02, M S_{\mathrm{e}}=0.052\right.$, n.s.], age interacted significantly with $\operatorname{RF}\left[F(2,87)=4.16, M S_{\mathrm{e}}=0.052\right.$, $p<.05]$. For young participants, priming was greater for low-RF (mean, .36; $S D, .15$ ) than for high-RF (mean, $.28 ; S D, .18)$ targets $[t(29)=2.02, p<.05]$. For middleaged participants, too, priming was greater for low-RF (mean, .30; SD, .23) than for high-RF (mean .22; SD, $.22)$ targets $[t(29)=2.08, p<.05]$. Older adults showed the opposite pattern, with greater priming for high-RF targets (mean, .28; $S D, .18$ ) than for low-RF targets (mean, $.21 ; S D, .17)$, although this difference was not significant $[t(29)=1.41, p=.17]$. The priming data are depicted in Figure 2.

Analyzed another way, LSD follow-up tests indicated that the three age groups obtained similar amounts of priming for high-frequency words (n.s.). In contrast, priming for low-frequency targets differed significantly across the age groups, with significantly greater priming for younger than for older participants $(p<.01)$ and marginally greater priming for middle-aged than for older participants $(p=.06)$. Young and middle-aged groups did not differ from one another $(p=.21)$.

Explicit WSC performance. The data were analyzed similarly to the implicit data, first analyzing baseline, followed by studied performance using a cued recall score corrected for baseline performance (see Table 5). At baseline, targets were more likely to be produced when they were high $\mathrm{RF}$ than when they were low $\operatorname{RF}[F(1,87)=$ $\left.38.80, M S_{\mathrm{e}}=0.037, p<.0001\right]$, and targets were more likely to be produced when they were sound matched than when they were mismatched $\left[F(1,87)=17.30, M S_{\mathrm{e}}=\right.$ $0.041, p<.0001]$. Age did not influence baseline probabilities $(F<1)$, and neither RF nor sound match interacted with age $\left(F_{\mathrm{S}}<1\right)$.

Corrected cued recall scores indicated that high-RF targets were recalled more readily than low-RF targets 


\section{Priming}

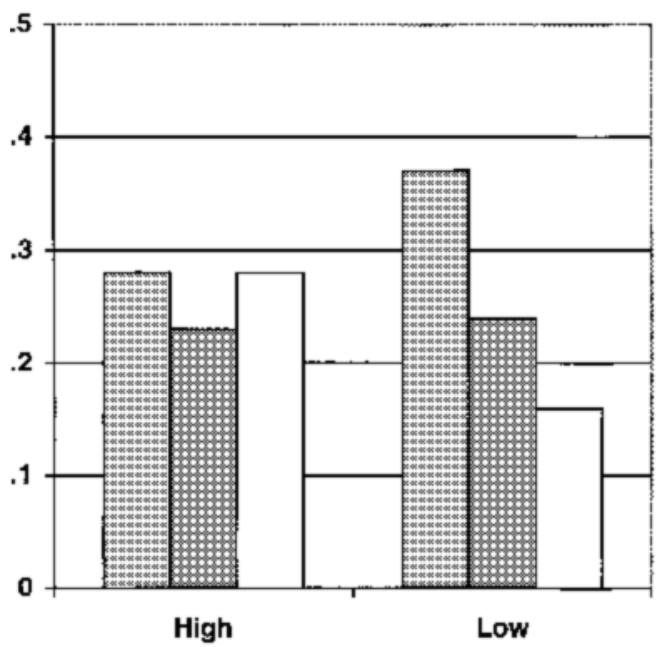

Cued recall

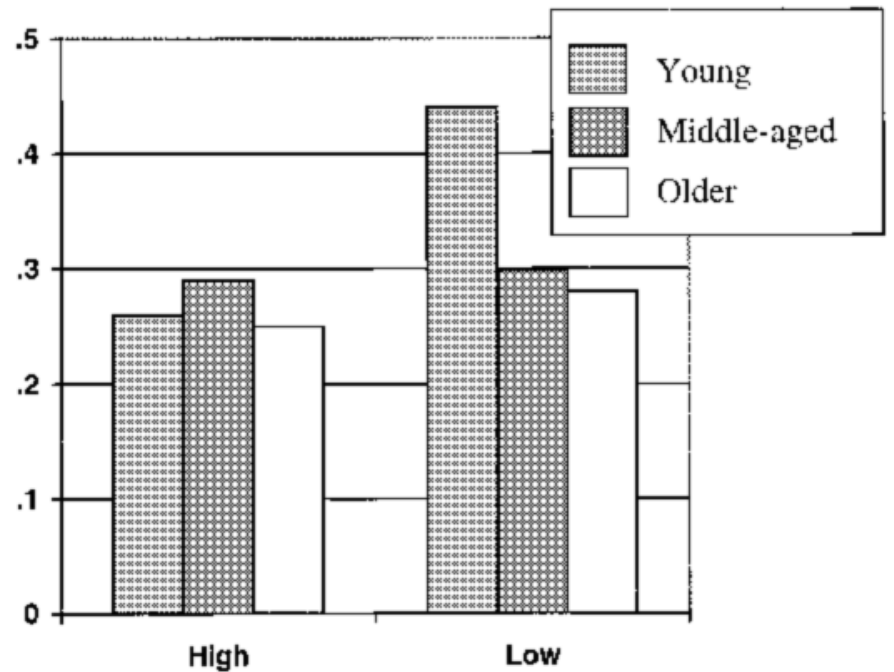

Figure 2. Word-stem completion results for priming and cued recall (corrected for baseline performance) for Experiment 2.

$\left[F(1,87)=5.44, M S_{\mathrm{e}}=0.078, p<.05\right]$. Although sound matched items were recalled more often than mismatched items (.33 vs. .27, respectively), this difference did not reach significance $\left[F(1,87)=1.94, M S_{\mathrm{e}}=0.072, p=\right.$ .17]. There was a marginal main effect of age $[F(2,87)=$ $\left.2.55, M S_{\mathrm{e}}=0.08, p=.08\right]$. However, age interacted with $\operatorname{RF}\left[F(2,87)=3.43, M S_{\mathrm{e}}=0.078, p<.05\right]$. Paired $t$ tests showed that young adults recalled more low- than high-RF items $[t(29)=3.21, p<.01]$, whereas middleaged and older adults recalled equal numbers of highand low-RF items (n.s.).

LSD follow-up tests indicated no differences for young, middle-aged, and older participants in cued recall for high-RF targets. However, young participants recalled more low-RF targets than did either middle-aged $(p<.01)$ or older $(p<.001)$ participants, whereas middle-aged and older participants did not differ from one another (see Figure 2).

\section{Discussion}

The results confirm earlier item analyses indicating that baseline and studied performance on WSC are independently influenced by relative familiarity and cuespecific matching, reflected in the pronunciation match between the stem and the target completion. Consistent with Experiment 1, age did not influence baseline probabilities or the utilization of familiarity and cue-specific matching at baseline. However, the degree to which RF predicted WSC priming and cued recall depended on the age group. Although all the age groups produced similar rates of high-RF responses, older adults produced and recalled fewer low-RF words. In contrast, cue-specific matching was employed similarly by young, middle-aged, and older adults in both priming and cued recall. The results may explain some of the inconsistencies in the WSC priming literature. Demonstrations of decreased priming in older adults will depend critically on the target materials. A case in point is Experiment 1, where two thirds of the materials were specifically chosen to have low first completion baseline probabilities. Retrospectively, we calculated the RF values for these targets and found an average RF of 7.8 (SD, 3.4), which is comparable with the low-RF targets used in Experiment 2 (mean, 7.2; SD, 3.1). It is not surprising, then, that we obtained significant declines in both priming and cued recall performance in our older participants in Experiment 1.

Finally, the performance of the middle-aged group on both priming and cued recall tended to fall somewhere midway between that of the young and that of the older participants, again consistent with Experiment 1, suggesting that age-related changes in WSC performance occur in the 4th and 5th decades, as well as during the 6th decade and beyond. This finding must be taken into account by models of age-related memory differences in WSC performance.

\section{GENERAL DISCUSSION}

Taken together, the results of the present study provide important information regarding the nature of WSC, as well as age-related differences in WSC performance.

\section{Search and Selection in Explicit and Implicit WSC}

Recent studies have highlighted the importance of word frequency (Postle \& Corkin, 1999) and phonological processing (Rueckl \& Mathew, 1999) in WSC. The present study extends these results, indicating that both factors independently contribute to the selection of a stem completion. Importantly, rank order word frequency and 
cue-specific matching affected baseline, priming, and cued recall similarly, suggesting some degree of overlap in search and selection processes for these tasks.

These results are inconsistent with the view of Winocur et al. (1996) that search is engaged only during priming. These authors argued that, when instructed to produce the first word that comes to mind, participants use the stem cue to search the lexicon for possible matches and that selection of an item is biased by its recent presentation. Awareness on the part of the participant that the stem cue is directly linked to a memory trace obviates the need for search. Instead, retrieval processes are invoked that provide direct and relatively automatic access to the matching trace. By this view, search-related factors influencing baseline and priming should not have parallel effects on WSC cued recall. More recently, Nyberg, Winocur, and Moscovitch (1997) have amended this view to suggest that WSC cued recall will utilize search processes when the stem does not overly constrain the completion set size (the total number of possible completions for a given stem).

Other views more consistent with the present results are those in which WSC priming and cued recall tasks incorporate similar retrieval processes but differ in the use of a postretrieval check of episodic appropriateness (Humphreys et al., 1989), or as Tulving (1983) described it, a postecphoric output decision. By these views, the critical difference between WSC priming and cued recall occurs after a completion is produced. During a priming task, once an item is produced, there is no need to reconsider that selection as long as it is a legitimate completion. When participants are required to recall previously encountered words, however, the decision regarding whether or not an item was previously encountered is based on other information, including the experimental context and the semantic associations made at the time of study. If the first completion is deemed inappropriate, another item is chosen and subjected to the postselection check, and so on. Whereas postselection checking is specific to explicit WSC, the production stage is common across implicit and explicit forms of WSC, and it is this stage that is most likely influenced by familiarity and cuespecific matching factors.

An important difference between familiarity and cuespecific factors is the degree to which they are unique to the test cue. The effect of familiarity as reflected in word frequency is well known to influence performance on many priming and recollective tasks (e.g., Glanzer \& Bowles, 1976; Jacoby \& Dallas, 1981). In contrast, cuespecific matching variables are uniquely related to a particular type of cue and will not generalize to other paradigms or, probably, even to slightly modified cues. A task such as reading a degraded word, for example, would reasonably be expected to rely predominantly on visual processing of letter shapes, whereas WSC relies more heavily on phonological processing, and so forth. Cuespecific matching adds an important caveat to models such as Humphreys et al. (1989), in which all part-word cues are considered as a unitary category that includes word stems, word endings, word fragments, and degraded visual words. Although there may indeed be similarities in the processing of part-word cues, the unique processes engaged for a given cue type may be equally, if not more, important in predicting performance. In addition, partword cues will engage search processes to varying degrees, depending on how the cue constrains the number of possible completions. Word fragments and anagrams often have only one or two solutions, whereas three-letter word stems may have as many as several hundred and twoletter stems will vastly increase this solution set (Nyberg et al., 1997).

\section{Models of WSC Performance in Older Adults}

Winocur et al. (1996) suggested that older individuals are impaired on WSC owing to inefficient use of a perceptual cue for lexical search. The present results are not consistent with this view, since older adults used the pronunciation of the stem to guide search to a similar degree as younger adults. However, the present results clearly indicated that there were large age-related word frequency differences in WSC priming and cued recall. These results are consistent with the view of Balota and Ferraro (1993, 1996; see also Hasher \& Zacks, 1988; Parkin, 1993), who focus on the increased reliance on familiar or automatic responses among older adults due to the loss of inhibitory control over partially activated information. Whereas younger adults produced and recalled distinctive, lower frequency words (Graf \& Ryan, 1990), older adults relied more heavily on the familiarity of the word in their choice of a completion. However, one aspect of the present results does not easily fit with the inhibitory control hypothesis. This view predicts that older adults should show the same bias toward high-frequency words during any task involving lexical search, regardless of whether or not some of the targets were recently encountered. The absence of a baseline effect suggests that age-related changes in performance have more to do with the impact of the single prior study episode, rather than a general change in lexical search. However, the present results probably reflect the insensitivity inherent in most baseline data. Since most participants produce only a few baseline target words, it is unlikely that subtle group differences can be detected. Balota and Ferraro (1996) found increased baseline word frequency effects in olderold adults on a lexical decision task, but they obtained 3,840 observations per age group in order to demonstrate this relatively subtle effect.

Finally, the performance of middle-aged adults consistently fell midway between that of younger and that of older adults in the present experiments, suggesting that word frequency sensitivity in older adults is altered through a lifetime of experience with language, rather than by a dysfunctional memory system. Presumably, frequency information continues to be updated throughout life, and thus it is not unreasonable to assume that frequency effects will vary as a result. In essence, although 
the organization of the lexicon may remain frequency based throughout life, it may settle over time into a structure that is less variable. The distinctive characteristic of low-frequency words is attenuated in older adults, whereas their ability to utilize phonologicallybased lexical search remains intact. Identifying the source of this attenuation effect will be crucial to understanding the mechanisms of memory changes associated with normal aging.

\section{REFERENCES}

Abbenhuis, M. A., Raajmakers, W. G. M., Raajmakers, J. G. W., \& VAN WoERdEN, G. J. M. (1990). Episodic memory in dementia of the Alzheimer type and in normal aging: Similar impairment in automatic processing. Quarterly Journal of Experimental Psychology, 42A, 569-583.

Balota, D. A., \& Chumbley, J. I. (1984). Are lexical decisions a good measure of lexical access? The role of word frequency in the neglected decision stage. Journal of Experimental Psychology: Human Perception \& Performance, 10, 340-357.

Balota, D. A., \& Ferraro, F. R. (1993). A dissociation of frequency and regularity effects in pronunciation performance across young adults, older adults, and individuals with senile dementia of the Alzheimer type. Journal of Memory \& Language, 32, 573-592.

Balota, D. A., \& Ferraro, F. R. (1996). Lexical, sublexical, and implicit memory processes in healthy young and healthy older adults and in individuals with dementia of the Alzheimer type. Neuropsychology, 10, 82-95.

Bowers, J. S., \& SCHACTER, D. L. (1990). Implicit memory and test awareness. Journal of Experimental Psychology: Learning, Memory, \& Cognition, 16, 404-416.

Chiarello, C., \& Hoyer, W. J. (1988). Adult age differences in implicit and explicit memory: Time course and encoding effects. Psychology \& Aging, 3, 358-366.

Davis, H. P., Cohen, A., Gandy, M., Colombo, P., VanDusseldorp, G., Simolke, N., \& Romano, J. (1990). Lexical priming deficits as a function of age. Behavioral Neuroscience, 104, 288-297.

Dick, M. B., KeAN, M. L., \& SANDS, D. (1989). Memory for internally generated words in Alzheimer-type dementia: Breakdown in encoding and semantic memory. Brain \& Cognition, 9, 88-108.

Fleischman, D. A., \& Gabrieli, J. D. E. (1998). Repetition priming in normal aging and Alzheimer's disease: A review of findings and theories. Psychology \& Aging, 13, 88-119.

Glanzer, M., \& Bowles, N. (1976). Analysis of the word frequency effect in recognition memory. Journal of Experimental Psychology: Human Learning \& Memory, 2, 21-31.

Graf, P., \& MAndler, G. (1984). Activation makes words more accessible, but not necessarily more retrievable. Journal of Verbal Learning \& Verbal Behavior, 23, 553-568.

GraF, P., \& RYAN, L. (1990). Transfer-appropriate processing for implicit and explicit memory. Journal of Experimental Psychology: Learning, Memory, \& Cognition, 16, 978-992.

Graf, P., \& Williams, D. (1987). Completion norms for 40 three-letter word stems. Behavior Research Methods, Instruments, \& Computers, 19, 422-445.

HASHER, L., \& ZACKS, R. T. (1988). Working memory, comprehension, and aging: A review and a new view. Psychology of Learning \& Motivation, 22, 193-225.

Hashtroudi, S., Chrosniak, L. D., \& Schwartz, B. L. (1991). Effects of aging on priming and skill learning. Psychology \& Aging, 6, 605-615.

Hintzman, D. L., \& Hartry, A. L. (1990). Item effects in recognition and fragment completion: Contingency relations vary for different subsets of words. Journal of Experimental Psychology: Learning, Memory, \& Cognition, 16, 955-968.

Howard, D. V. (1988). Implicit and explicit assessment of cognitive aging. In M. L. Howe \& C. J. Brainerd (Eds.), Cognitive development in adulthood: Progress in cognitive development research (pp. 3-37). New York: Springer-Verlag.
Hultsch, D. F., Masson, M. E. J., \& Small, B. J. (1991). Adult age differences in direct and indirect tests of memory. Journal of Gerontology: Psychological Sciences, 46, 22-30.

Humphreys, M. S., Bain, J. D., \& Pike, R. (1989). Different ways to cue a coherent memory system: A theory for episodic, semantic, and procedural tasks. Psychological Review, 96, 208-233.

JАСОВY, L. L. (1991). A process dissociation framework: Separating automatic from intentional uses of memory. Journal of Memory \& Language, 30, 513-541.

JACOBY, L. L., \& DALlas, M. (1981). On the relationship between autobiographical memory and perceptual learning. Journal of Experimental Psychology: General, 110, 300-340.

JaVA, R. I., \& Gardiner, J. M. (1991). Priming and aging: Further evidence of preserved memory function. American Journal of Psychology, 104, 89-100.

Jernigan, T. L., \& Ostergaard, A. L. (1993). Word priming and recognition memory are both affected by mesial temporal lobe damage. Neuropsychology, 7, 14-26.

Johnson, M. K., \& HASHER, L. (1987). Human learning and memory. Annual Review of Psychology, 38, 631-668.

Karayanidis, F., Andrews, S., Ward, P. B., \& McConaghy, N. (1993). Event-related potentials and repetition priming in young, middleaged and elderly normal subjects. Cognitive Brain Research, 1, 123-134

KuČERA, H., \& Francis, W. (1967). Computationalanalysis of presentday American English. Providence, RI: Brown University Press.

La Voie, D., \& Light, L. L. (1994). Adult age differences in repetition priming: A meta-analysis. Psychology \& Aging, 9, 539-553.

Light, L. L., \& Kennison, R. F. (1996). Guessing strategies, aging, and bias effects in perceptual identification. Consciousness \& Cognition, 5, 463-499.

Light, L. L., \& LaVoie, D. (1993). Direct and indirect measures of memory in old age. In P. Graf \& M. E. J. Masson (Eds.), Implicit memory: New directions in cognition, development, and neuropsychology (pp. 207-230). Hillsdale, NJ: Erlbaum.

Light, L. L., La Voie, D., Valencia-Laver, D., Albertson-Owens, S. A., \& MeAD, G. (1992). Direct and indirect measures of memory for modality in young and older adults. Journal of Experimental Psychology: Learning, Memory, \& Cognition, 18, 1284-1297.

Light, L. L., \& SingH, A. (1987). Implicit and explicit memory in young and older adults. Journal of Experimental Psychology: Learning, Memory, \& Cognition, 13, 531-541.

Light, L. L., Singh, A., \& CAPPS, J. L. (1986). Dissociation of memory and awareness in young and older adults. Journal of Clinical \& Experimental Neuropsychology, 8, 62-74.

Nelson, D. L., Canas, J. J., Bajo, M. T., \& Keelean, P. D. (1987), Comparing word fragment completion and cued recall with letter cues. Journal of Experimental Psychology: Learning, Memory, \& Cognition, 13, 542-552.

Nyberg, L., Winocur, G., \& Moscovitch, M. (1997). Correlation between frontal lobe functions and explicit and implicit stem completion in healthy elderly. Neuropsychology, 11, 70-77.

OSTERGAARD, A. L. (1994). Dissociations between word priming effects in normal subjects and patients with memory disorders: Multiple memory systems or retrieval? Quarterly Journal of Experimental Psychology, 47A, 331-364.

OstergaARD, A. L. (1998). The effects on priming of word frequency, number of repetitions, and delay depend on the magnitude of priming. Memory \& Cognition, 26, 40-60.

OstergaARD, A. L. (1999). Priming deficits in amnesia: Now you see them, now you don't. Journal of the International Neuropsychological Society, 5, 175-190.

PARK, D. C., \& Shaw, R. J. (1992). Effect of environmental support on implicit and explicit memory in younger and older adults. Psychology \& Aging, 7, 632-642.

PARKIN, A. J. (1993). Implicit memory across the lifespan. In P. Graf \& M. E. J. Masson (Eds.), Implicit memory: New directions in cognition, development, and neuropsychology (pp. 207-230). Hillsdale, NJ: Erlbaum.

Postle, B. R, \& CoRkin, S. (1999). Manipulation of familiarity reveals a necessary lexical component of the word-stem completion priming effect. Memory \& Cognition, 27, 12-25. 
Richardson-Klavehn, A., \& BJork, R. A. (1988). Measures of memory. Annual Review of Psychology, 39, 475-543.

Roediger, H. L., III, Weldon, M. S., Stadler, M. A., \& Riegler, G. H. (1992). Direct comparison of word stems and word fragments in implicit and explicit retention tests. Journal of Experimental Psychology: Learning, Memory, \& Cognition, 18, 1251-1269.

RUECKL, J. G., \& MATHEW, S. (1999). Implicit memory for phonological processes in visual stem completion. Memory \& Cognition, 27, 1-11.

RYBASH, J. M. (1994). Aging, associative priming and test awareness. Aging \& Cognition, 1, 158-173.

Scarborough, D. L., Cortese, C., \& Scarborough, H. S. (1977). Frequency and repetition effects in lexical memory. Journal of Experimental Psychology: Human Perception \& Performance, 3, 1-17.

Schacter, D. L., Church, B., \& Osowiecki, D. M. (1994). Auditory priming in elderly adults: Impairment of voice-specific implicit memory. Memory, 2, 295-323.
Small, B. J., Hultsch, D. F., \& Masson, M. E. J. (1995). Adult age differences in perceptually based, but not conceptually based implicit tests of memory. Journal of Gerontology: Psychological Sciences, 50B, 162-170.

Squire, L. R., Shimamura, A. P., \& GRaf, P. (1987). Strength and duration of priming effects in normal subjects and amnesic patients. Neuropsychologia, 25, 195-210.

Tulving, E. (1983). Elements of episodic memory. Oxford: Oxford University Press, Clarendon Press.

Winocur, G., Moscovitch, M., \& Stuss, D. T. (1996). Explicit and implicit memory in the elderly: Evidence for double dissociation involving medial temporal- and frontal-lobe functions. Neuropsychology, 10, 57-65.

(Manuscript received July 29, 1998; revision accepted for publication February 27, 2001.) 